\title{
Concordance group and stable commutator length in braid groups
}

\author{
MiCHAEL BRANDENBURSKY \\ JAREK KĘDRA
}

\begin{abstract}
We define quasihomomorphisms from braid groups to the concordance group of knots and examine their properties and consequences of their existence. In particular, we provide a relation between the stable four ball genus in the concordance group and the stable commutator length in braid groups, and produce examples of infinite families of concordance classes of knots with uniformly bounded four ball genus. We also provide applications to the geometry of the infinite braid group $\mathbf{B}_{\infty}$. In particular, we show that the commutator subgroup $\left[\mathbf{B}_{\infty}, \mathbf{B}_{\infty}\right]$ admits a stably unbounded conjugation invariant norm. This answers an open problem posed by Burago, Ivanov and Polterovich.
\end{abstract}

20F36, 57M25; 20F69

\section{Introduction and presentation of the results}

We define maps $\Psi_{n}: \mathbf{B}_{n} \rightarrow \operatorname{Conc}\left(\mathbf{S}^{3}\right)$ from the braid groups to the concordance group of knots in the three-dimensional sphere and observe that they have good algebraic and geometric properties. The maps are defined by closing a braid appropriately to obtain a knot and then take its concordance class. They are compatible with inclusions $\mathbf{B}_{n} \rightarrow \mathbf{B}_{n+1}$ and hence they induce a well-defined map $\Psi_{\infty}: \mathbf{B}_{\infty} \rightarrow \operatorname{Conc}\left(\mathbf{S}^{3}\right)$ on the infinite braid group.

We examine the geometric properties with respect to the conjugation invariant word norm on the braid group and the norm defined by the four ball genus on the concordance group. The first observation is that the maps $\Psi_{n}$ are quasihomomorphisms with the defects depending on $n$ and the second is that the maps are Lipschitz with the same Lipschitz constant independent on $n$. The latter implies that the map defined on the infinite braid group is also Lipschitz.

Before providing more details let us highlight several applications of these results.

- We relate the stable commutator length on braid groups and the stable four ball genus (Corollary 1.5) which answers a question of Livingston [10] (who attributes the question to D Calegari). 
- We provide an easy way of constructing infinite families of knots with uniformly bounded four ball genus (Corollary 1.6 and the subsequent examples).

- We construct infinite families of prime knots with unbounded stable four ball genus (Corollary 1.9).

- We prove that the commutator subgroup of the infinite braid group admits a stably unbounded conjugation invariant norm. This result is interesting because this (perfect) group admits no nontrivial quasimorphisms (see Kotschick [8]) (the main tool for proving stable unboundedness) and its commutator length is bounded [3]. This answers an open problem from the paper by Burago, Ivanov and Polterovich [3]. Another solution for the same problem has been recently provided by Kawasaki [7] who showed that the group of compactly supported symplectic diffeomorphisms of the Euclidean space is stably unbounded.

In the remaining part of the introduction we state our results and provide more details.

\section{Two main observations}

Let $A$ be an abelian group equipped with a pseudonorm and let $|a|$ denote the value of the pseudonorm on an element $a \in A$. Let $G$ be a group. A map $\psi: G \rightarrow A$ is called a quasihomomorphism if there exist a constant $K_{\psi} \geq 0$ such that

$$
|\psi(g)-\psi(g h)+\psi(h)| \leq K_{\psi}
$$

for all $g, h \in G$. The infimum of all such $K_{\psi}$ is called the defect of $\psi$ and is denoted by $D_{\psi}$. A real valued quasihomomorphism is traditionally called a quasimorphism.

Let ( ): $\mathbf{B}_{n} \rightarrow \Sigma_{n}$ be the natural projection from the braid group to the symmetric group. Let $\mathbf{B}_{n}^{K}$ denote the subset consisting of braids whose closures are knots. We define a projection $\pi_{n}: \mathbf{B}_{n} \rightarrow \mathbf{B}_{n}^{K}$ by sending a braid $\alpha$ to the braid $\alpha \sigma_{(\alpha)}$, where the braid $\sigma_{(\alpha)}$ depends only on the permutation induced by $\alpha$ (see Section 2.5).

Let $\operatorname{Conc}\left(\mathbf{S}^{3}\right)$ denote the group of smooth concordance classes of knots in the threedimensional sphere. It is equipped with a norm defined by the four ball genus (see Section 2 for details). Let $\Psi_{n}: \mathbf{B}_{n} \rightarrow \operatorname{Conc}\left(\mathbf{S}^{3}\right)$ be defined as the composition

$$
\mathbf{B}_{n} \stackrel{\pi_{n}}{\longrightarrow} \mathbf{B}_{n}^{K} \stackrel{\text { closure }}{\longrightarrow} \text { Knots } \stackrel{[-]}{\longrightarrow} \operatorname{Conc}\left(\mathbf{S}^{3}\right), \quad \Psi_{n}(\alpha):=\left[\widehat{\alpha \sigma_{(\alpha)}}\right],
$$

where $\widehat{\alpha}$ denotes the closure of the braid $\alpha$. Our first observation was essentially proven by the first author in [1]:

Theorem 1.1 The map $\Psi_{n}: \mathbf{B}_{n} \rightarrow \operatorname{Conc}\left(\mathbf{S}^{3}\right)$ is a quasihomomorphism with respect to the four ball genus norm and with defect $D_{\Psi_{n}} \leq 3 n+1$. Its image contains all concordance classes represented by knots which are closures of braids on $n$ strings. 
Remark The quasihomomorphisms $\Psi_{n}$ are compatible with the inclusions $\mathbf{B}_{n} \rightarrow$ $\mathbf{B}_{n+1}$ and hence the above construction defines a surjective map $\Psi_{\infty}: \mathbf{B}_{\infty} \rightarrow \operatorname{Conc}\left(\mathbf{S}^{3}\right)$ (see Section 2.5). However, using the fact that every homogeneous quasimorphism on $\mathbf{B}_{\infty}$ must be a homomorphism, see Kotschick [8], we show that the map $\Psi_{\infty}$ can't be a quasihomomorphism (Proposition 3.6).

Convention It is always assumed that $n$ in the notation $\mathbf{B}_{n}$ for the braid group is a natural number. Some of our statements extend to the infinite braid group. In such cases we emphasize that $n \in \mathbf{N} \cup\{\infty\}$.

Let $\sigma_{1}, \ldots, \sigma_{n-1} \in \mathbf{B}_{n}$ be the standard Artin generators of the braid group. That is, the braid $\sigma_{i}$ swaps the $i^{\text {th }}$ and the $(i+1)^{\text {st }}$ string. Observe that these braids are pairwise conjugate thus $\mathbf{B}_{n}$ is normally generated by the symmetric set $\left\{\sigma_{1}^{ \pm 1}\right\}$, where $n \in \mathbf{N} \cup\{\infty\}$. Let us consider the associated conjugation invariant word norm on $\mathbf{B}_{n}$, denoted by $\|\alpha\|$, and the induced biinvariant metric defined by $\mathrm{d}(\alpha, \beta):=\left\|\alpha \beta^{-1}\right\|$.

Theorem 1.2 Let $n \in \mathbf{N} \cup\{\infty\}$. The map $\Psi_{n}: \mathbf{B}_{n} \rightarrow \operatorname{Conc}\left(\mathbf{S}^{3}\right)$ is Lipschitz with respect to the conjugation invariant word norm on the braid group and the four ball genus norm on the concordance group. More precisely,

$$
\mathrm{g}_{4}\left(\Psi_{n}(\alpha)\right) \leq \frac{1}{2}\|\alpha\|
$$

for all braids $\alpha \in \mathbf{B}_{n}$.

Remark (1) It follows from Theorem 1.1 that $\Psi_{n}$ is Lipschitz with constant bounded above by the defect $D_{\Psi_{n}}$. We get a smaller constant in the above theorem by a more direct and elementary geometric argument.

(2) If one defines a metric on the concordance group by

$$
\mathrm{d}_{4}(K, L):=\mathrm{g}_{4}(K-L)
$$

then it follows from the above theorems that

$$
\mathrm{d}_{4}\left(\Psi_{n}(\alpha), \Psi_{n}(\beta)\right) \leq \frac{1}{2} \mathrm{~d}(\alpha, \beta)+D_{\Psi_{n}} .
$$

That is, the map $\Psi_{n}$ is large scale Lipschitz with respect to the metrics and for any natural number $n \in \mathbf{N}$.

Let us discuss applications and consequences of the above theorems. 


\section{Quasimorphisms on braid groups}

Composing the quasihomomorphism $\Psi_{n}$ with a suitable quasimorphism defined on the concordance group yields a quasimorphism on the braid group. More precisely, we have the following observation.

Corollary 1.3 Let $\varphi$ : $\operatorname{Conc}\left(\mathbf{S}^{3}\right) \rightarrow \mathbf{R}$ be a quasimorphism. If $\varphi$ is Lipschitz with respect to the four ball genus norm then the composition $\varphi \circ \Psi_{n}: \mathbf{B}_{n} \rightarrow \mathbf{R}$ is a quasimorphism.

This idea was used by the first author in [1]. The next applications provide new results.

\section{The quasihomomorphism $\Psi_{n}$ is Lipschitz with respect to the commutator length}

The commutator length $\operatorname{cl}(g)$ of an element $g$ in $[G, G]$ is defined to be the minimal number of commutators in $G$ whose product is equal to $g$. The following result is an application of Theorem 1.1 and a general fact about quasihomomorphisms presented in Lemma 3.2.

Corollary 1.4 The restriction of the quasihomomorphism $\Psi_{n}$ to the commutator subgroup $\left[\mathbf{B}_{n}, \mathbf{B}_{n}\right]$ is Lipschitz with respect to the commutator length. More precisely,

$$
\mathrm{g}_{4}\left(\Psi_{n}(\alpha)\right) \leq 4 D_{\Psi_{n}} \mathrm{cl}(\alpha)
$$

for any $\alpha \in\left[\mathbf{B}_{n}, \mathbf{B}_{n}\right]$.

The above result does not extend to the infinite case because the commutator length is bounded by 2 on the infinite braid group according to Burago, Ivanov and Polterovich (see Theorem 1.11).

\section{A relation between the stable four ball genus and the scl}

Livingston asked in [10, Section 8.1] whether there is a connection between the stable commutator length in groups and the stable four ball genus in $\operatorname{Conc}\left(\mathbf{S}^{3}\right)$. The next corollary provides such a connection.

Corollary 1.5 If $\alpha \in\left[\mathbf{B}_{n}, \mathbf{B}_{n}\right]$ then

$$
\operatorname{sg}_{4}\left(\Psi_{n}(\alpha)\right) \leq 4 D_{\Psi_{n}} \operatorname{scl}(\alpha)+D_{\Psi_{n}} .
$$

In particular, if the stable commutator length of $\alpha$ is trivial then the stable four ball genus of $\Psi_{n}(\alpha)$ is bounded above by the defect $D_{\Psi_{n}}$ :

$$
\operatorname{scl}(\alpha)=0 \quad \Longrightarrow \quad \operatorname{sg}_{4}\left(\Psi_{n}(\alpha)\right) \leq D_{\Psi_{n}} .
$$


Remark The braids $\alpha^{2 n}$ from Example 4.4 have trivial stable commutator length and $\operatorname{sg}_{4}\left(\Psi_{4}\left(\alpha^{2 n}\right)\right)>0$. The last inequality follows from the fact that the $\Psi_{4}\left(\alpha^{2 n}\right)=$ $\left[T_{2 n+1} \# T_{2 n-1}^{*}\right]$, where $T_{k}$ is the torus knot obtained by closing the braid $\sigma_{1} \in \mathbf{B}_{2}$ and $T_{k}^{*}$ is its mirror image. The signature of $T_{2 n+1} \# T_{2 n-1}^{*}$ is equal to two and hence its stable four ball genus is bounded from below by one, due to the Murasugi inequality (4-1).

\section{Families of knots with uniformly bounded four ball genus}

The next result can be used to produce concrete infinite families of knots (and concordance classes) with uniformly bounded four ball genus.

Corollary 1.6 Let $\alpha \in\left[\mathbf{B}_{n}, \mathbf{B}_{n}\right]$. If $\operatorname{scl}(\alpha)=0$ then the concordance classes $\Psi_{n}\left(\alpha^{k}\right)$ for $k \in \mathbf{Z}$ have uniformly bounded four ball genus.

Remark Infinite families of knots with bounded four ball genus have been known since the 1960s [9, Section 3.1]. Since it is easy to provide braids with trivial stable commutator length, our corollary yields many families of knots for which checking the boundedness of the four ball genus could be difficult otherwise.

Examples of braids with trivial stable commutator length abound. For instance, a braid which is conjugate to its inverse has trivial stable commutator length.

Example 1.7 Let $\alpha=\sigma_{1} \sigma_{2}^{-1} \in \mathbf{B}_{3}$. It is straightforward to see that $\Delta \alpha \Delta^{-1}=\alpha^{-1}$, where $\Delta=\sigma_{1} \sigma_{2} \sigma_{1}$ is the Garside element. Consequently, $\operatorname{scl}(\alpha)=0$ and it follows from Corollary 1.6 that the family consisting of the closures of the braids $\alpha^{k} \sigma_{\left(\alpha^{k}\right)}$ has uniformly bounded four ball genus. It is not difficult to show that this family is infinite. It is easy to show that the closure of the braid $\alpha^{k} \sigma_{\left(\alpha^{k}\right)}$ is an amphicheiral knot and hence the concordance class of $\Psi_{3}\left(\alpha^{k}\right)$ is of order at most two in $\operatorname{Conc}\left(\mathbf{S}^{3}\right)$. However, it remains an open question whether the family $\Psi_{3}\left(\alpha^{k}\right)$ of concordance classes is infinite.

Example 1.8 Let $\alpha=\sigma_{1} \sigma_{3}^{-1} \in \mathbf{B}_{4}$. It is again straightforward to see that this braid is conjugate to its inverse and hence it has trivial stable commutator length. However, in this case we obtain that the set of concordance classes $\Psi\left(\alpha^{2 n}\right)$ is infinite (Section 4.6) and has uniformly bounded four ball genus. 


\section{Families of prime knots with unbounded stable four ball genus}

Let $G$ be a group and $\varphi: G \rightarrow \mathbf{R}$ be a quasimorphism. We denote by $\bar{\varphi}: G \rightarrow \mathbf{R}$ the homogenization of $\varphi$, ie $\bar{\varphi}(g):=\lim _{p \rightarrow \infty} \varphi\left(g^{p}\right) / p$. For more information about quasimorphisms, see Calegari [4].

Corollary 1.9 Let $\varphi$ : $\operatorname{Conc}\left(\mathbf{S}^{3}\right) \rightarrow \mathbf{R}$ be a quasimorphism which is Lipschitz with respect to the four ball genus norm. Let $C_{\varphi}$ denote its Lipschitz constant. If $\alpha \in \mathbf{B}_{n}$ and $p \in \mathbf{N}$, then

$$
\operatorname{sg}_{4}\left(\Psi_{n}\left(\alpha^{p}\right)\right) \geq \frac{\left|\left(\overline{\varphi \circ \Psi_{n}}\right)(\alpha)\right|}{C_{\varphi}} \cdot p-D_{\Psi_{n}}
$$

where $\overline{\varphi \circ \Psi_{n}}$ denotes the homogenization of the quasimorphism $\varphi \circ \Psi_{n}$. If particular, if the quasimorphism $\varphi \circ \Psi_{n}$ is unbounded on the cyclic subgroup generated by $\alpha$ then the stable genus of the knots $\Psi_{n}\left(\alpha^{p}\right)$ grows linearly with $p$.

Example 1.10 Let $\varphi$ : $\operatorname{Conc}\left(\mathbf{S}^{3}\right) \rightarrow \mathbf{R}$ be a quasimorphism given by the signature of a knot. It is known (see Murasugi [13]) that it is Lipschitz with respect to the four ball genus and hence we can apply the above corollary. In this example we show that there exists a braid $\alpha \in \mathbf{B}_{3}$, such that for each $p \in \mathbf{N}$ the knot $\Psi_{3}\left(\alpha^{p}\right)$ is prime and the composition $\varphi \circ \Psi_{3}$ is unbounded on the cyclic subgroup generated by $\alpha$. The braid $\alpha$ is given by the following presentation $\alpha=\sigma_{1}^{-4} \sigma_{2} \sigma_{1}^{2} \sigma_{2} \in \mathbf{B}_{3}$. The fact that $\Psi_{3}\left(\alpha^{p}\right)$ is a prime knot for each $p \in \mathbf{N}$ follows from Morton [12].

\section{Applications to conjugation invariant geometry of the infinite braid group}

Recall that a norm $v$ on a group $G$ is called stably unbounded if there exists $g \in G$ such that

$$
s v(g)=\lim _{p \rightarrow \infty} \frac{v\left(g^{p}\right)}{p} \neq 0 .
$$

If $\psi: G \rightarrow \mathbf{R}$ is a nontrivial homogeneous quasimorphism which is Lipschitz with respect to $v$ then $v$ is stably unbounded. This is the usual argument proving the stable unboundedness of a norm.

It follows from a result of Kotschick [8] that the only nontrivial homogeneous quasimorphism on the infinite braid group is the abelianization (up to a constant). Moreover, the commutator length on $\left[\mathbf{B}_{\infty}, \mathbf{B}_{\infty}\right]$ is bounded by two according to Burago, Ivanov and Polterovich [3, Theorem 2.2]. More precisely, they proved the following result. 
Theorem 1.11 (Burago, Ivanov and Polterovich) Let $H$ be a subgroup of a group $G$. Suppose that for every natural number $m \in \mathbf{N}$ there exists an element $g \in G$ such that the conjugate subgroups $\mathrm{g}^{i} \mathrm{Hg}^{-i}$ and $\mathrm{g}^{j} \mathrm{Hg}^{-j}$ pairwise commute for $0 \leq i<j \leq m$. Then the commutator length in $G$ of every element $h \in H$ is bounded by $t w o: \operatorname{cl}_{G}(h) \leq 2$.

Observe that the hypothesis of the above theorem is satisfied by the braid groups $\mathbf{B}_{n} \subset \mathbf{B}_{\infty}$ for every $n \in \mathbf{N}$ (see the proof of Proposition 3.10 for a detailed argument). This implies that the commutator length on the infinite braid group is bounded by two.

On the other hand, the diameter of the infinite braid group with respect to the biinvariant word metric is infinite. To see this consider the projection $\mathbf{B}_{\infty} \rightarrow \Sigma_{\infty}$ to the infinite symmetric group. It is Lipschitz and the cardinality of the support of a permutation defines a conjugation invariant norm on the symmetric group which is clearly unbounded. This implies that the conjugation invariant word norm is unbounded on the infinite braid group. The argument, however, says nothing on the geometry of cyclic subgroups of the infinite braid group and, in particular, it does not answer the question whether the word norm is stably unbounded. Our next corollary answers this question affirmatively.

Corollary 1.12 Let $\alpha \in\left[\mathbf{B}_{\infty}, \mathbf{B}_{\infty}\right]$. If there exists a Lipschitz quasimorphism

$$
\varphi: \operatorname{Conc}\left(\mathbf{S}^{3}\right) \rightarrow \mathbf{R}
$$

such that $\varphi\left(\Psi_{\infty}(\alpha)\right) \neq 0$ then

$$
\lim _{p \rightarrow \infty} \frac{\left\|\alpha^{p}\right\|}{p}>0
$$

In particular, the braid $\sigma_{1}^{-4} \sigma_{2} \sigma_{1}^{2} \sigma_{2} \in\left[\mathbf{B}_{\infty}, \mathbf{B}_{\infty}\right]$ discussed in Example 1.10 satisfies the above assumption and hence the conjugation invariant word norm on $\mathbf{B}_{\infty}$ is stably unbounded.

Burago, Ivanov and Polterovich [3] posed several problems about existence of groups with certain metric properties. One of them asks if there exists a group $G$ with the following properties:

(1) $G$ has finite abelianization.

(2) The commutator length of $G$ is stably trivial.

(3) $G$ admits a stably unbounded conjugation invariant norm.

The infinite braid group satisfies the last two conditions of the above problem but its abelianization is infinite cyclic. We have, however, the following observation. 
Theorem 1.13 The commutator subgroup $\left[\mathbf{B}_{\infty}, \mathbf{B}_{\infty}\right]$ of the infinite braid group satisfies the conditions of the above problem.

Proof Observe that the commutator subgroup $\left[\mathbf{B}_{\infty}, \mathbf{B}_{\infty}\right]$ is the union of the commutator subgroups $\left[\mathbf{B}_{n}, \mathbf{B}_{n}\right]$ of the braid group on finitely many strings. Let us justify that the group $\left[\mathbf{B}_{\infty}, \mathbf{B}_{\infty}\right]$ satisfies the properties of the above problem.

(1) It is known, see Gorin and Lin [6], that the commutator subgroup $\left[\mathbf{B}_{n}, \mathbf{B}_{n}\right]$ of the braid group is perfect for $n>4$. This implies that the group $\left[\mathbf{B}_{\infty}, \mathbf{B}_{\infty}\right]$ is perfect as well. Equivalently, its abelianization is trivial.

(2) Observe that the subgroups $\left[\mathbf{B}_{n}, \mathbf{B}_{n}\right] \subset\left[\mathbf{B}_{\infty}, \mathbf{B}_{\infty}\right]$ satisfy the assumption of Theorem 1.11 (Proposition 3.10). This implies that the commutator length is bounded by two and, in particular, it is stably trivial.

(3) The restriction of the conjugation invariant word norm from the whole infinite braid group to its commutator subgroup is stably unbounded due to Corollary 1.12.

Acknowledgements We would like to thank Steve Boyer, Ana Garcia Lecuona, Paolo Lisca and Brendan Owens for useful conversations, and Michał Marcinkowski for simplifying our proof of Corollary 1.5.

We are grateful to the anonymous referee for suggesting numerous improvements of the paper.

The first author was partially supported by the CRM-ISM fellowship. He would like to thank CRM-ISM Montreal for the support and great research atmosphere. Part of this work has been done during the authors stay at Max Planck Institute for Mathematics in Bonn. We wish to express our gratitude to the Institute for the support and excellent working conditions.

\section{Preliminaries}

\subsection{A norm on a group}

Let $G$ be a group. A function $v: G \rightarrow \mathbf{R}$ is called a pseudonorm if it satisfies the following conditions for all $g, h \in G$ :

(1) $v(g) \geq 0$.

(2) $v(g)=v\left(g^{-1}\right)$.

(3) $v(g h) \leq v(g)+v(h)$.

If in addition $v(g)=0$ if and only if $g=1_{G}$, then $v$ is called a norm. If $v\left(g h g^{-1}\right)=$ $v(h)$, then $v$ is called conjugation invariant. 
Remark If $G$ is an abelian group then a norm is often required to be homogeneous. That is, $v(n g)=|n| v(g)$ for all $g \in G$ and all integers $n \in \mathbf{Z}$. We do not make this requirement here.

The stabilization of $v$ is defined by

$$
s v(g):=\lim _{k \rightarrow \infty} \frac{v\left(g^{k}\right)}{k} .
$$

The stabilization of a norm does not have to be a pseudonorm. Both the nontriviality and the triangle inequality can be violated. If $G$ is abelian, however, then the stabilization of a norm is a pseudonorm. A norm $v$ is called stably unbounded if there exists $g \in G$ such that $s v(g) \neq 0$. Such an element $g$ is called undistorted with respect to the norm $v$.

\subsection{The conjugation invariant word norm}

Let $G$ be a normally finitely generated group. This means that there exists a finite symmetric set $S \subset G$ such that the set $S_{\text {conj }}$ of all conjugates of the elements of $S$ generates the group $G$. We also say that $S$ normally generates $G$. The associated word norm is defined by

$$
\|g\|:=\min \left\{k \in \mathbf{N} \mid g=s_{1} \cdots s_{k}, \text { where } s_{i} \in S_{\text {conj }}\right\} .
$$

This norm is, by definition, conjugation invariant and hence the induced metric, defined by $d_{S}(g, h):=\left\|g h^{-1}\right\|$ is biinvariant. The standard argument shows that any homomorphism $G \rightarrow H$ is Lipschitz with respect to $d_{S}$ and any biinvariant metric on $H$. Let us recall this basic argument.

Let $S$ be a finite symmetric set such that the conjugates of its elements generate the group $G$. Let $H$ be a group equipped with a conjugation invariant norm $v$ and let $\Psi: G \rightarrow H$ be a homomorphism. Let $g \in G$ be such that $\|g\|=k$ and let $C_{\Psi}:=\max \{v(\Psi(s)) \mid s \in S\}$. For any $h, f \in G$ let $f^{h}:=h f h^{-1}$. Observe that

$$
v(\Psi(g))=v\left(\Psi\left(s_{1}^{p_{1}} \ldots s_{k}^{p_{k}}\right)\right) \leq \sum_{i=1}^{k} v\left(\Psi\left(s_{i}^{p_{i}}\right)\right) \leq \sum_{i=1}^{k} v\left(\Psi\left(s_{i}\right)\right) \leq k C_{\Psi}=C_{\Psi}\|g\| .
$$

In particular, applying this to $\Psi$ being the identity homomorphisms, we obtain that the Lipschitz class of the norm \|\| does not depend on the choice of a finite set normally generating $G$. 


\subsection{The commutator length}

Let $g \in[G, G]$. Its commutator length is defined by

$$
\operatorname{cl}(g):=\min \left\{k \in \mathbf{N} \mid g=\left[a_{1}, b_{1}\right] \cdots\left[a_{k}, b_{k}\right], \text { where } a_{i}, b_{i} \in G\right\} .
$$

This quantity has been extensively studied and we refer the reader to D Calegari's book [4] for more information. The stable commutator length of an element $g$ is denoted by $\operatorname{scl}(g)$. Let us explain that for a braid group $\mathbf{B}_{n}$ the vanishing of the stable commutator length is equivalent to the vanishing of the stabilization of the conjugation invariant word norm.

It is known that braid groups satisfy the bq-dichotomy; see [2, Theorem 5.E]. This means that for every element $\alpha \in \mathbf{B}_{n}$ the cyclic subgroup $\langle\alpha\rangle$ is either biinvariantly bounded or there exists a homogeneous quasimorphism $q: G \rightarrow \mathbf{R}$ such that $q(\alpha) \neq 0$. If $\alpha \in\left[\mathbf{B}_{n}, \mathbf{B}_{n}\right]$ then the bq-dichotomy implies that the stable commutator length is trivial if and only if $\alpha$ generates a bounded cyclic subgroup. Consequently the stable commutator length of $\alpha$ is trivial if and only if the stable biinvariant word norm of $\alpha$ is trivial.

Remark The commutator subgroup of the infinite braid group contains undistorted elements, according to Corollary 1.12. Since $\mathbf{B}_{\infty}$ does not admit nontrivial quasimorphisms different from homomorphisms it does not satisfy the bq-dichotomy.

\subsection{The four ball genus norm on the concordance group}

Let $\operatorname{Conc}\left(\mathbf{S}^{3}\right)$ denote the abelian group of smooth concordance classes of knots in $\mathbf{S}^{3}$. Two oriented knots $K_{0}, K_{1} \in \mathbf{S}^{3}=\partial \mathbf{B}^{4}$ are concordant if there exists a smooth embedding $c: \mathbf{S}^{1} \times[0,1] \rightarrow \mathbf{B}^{4}$ such that $c\left(\mathbf{S}^{1} \times\{0\}\right)=K_{0}$ and $c\left(\mathbf{S}^{1} \times\{1\}\right)=-K_{1}$. The knot is called slice if it is concordant to the unknot. The addition in $\operatorname{Conc}\left(\mathbf{S}^{3}\right)$ is defined by the connected sum of knots. The inverse of an element $[K] \in \operatorname{Conc}\left(\mathbf{S}^{3}\right)$ is represented by the knot $-K^{*}$, where $-K^{*}$ denotes the mirror image of the knot $K$ with the reversed orientation. This group is equipped with a norm defined by the four ball genus. More precisely, $\mathrm{g}_{4}[K]$ is the minimal genus of an embedded oriented surface in $\mathbf{B}^{4}$ bounded by the knot $K$. We will call it the four ball genus norm. Its stabilization is denoted by $\operatorname{sg}_{4}[K]$. For more information about the group $\operatorname{Conc}\left(\mathbf{S}^{3}\right)$, see [9].

\subsection{The knot closure of a braid and the definition of $\Psi_{n}$}

Let $\mathbf{B}_{n}$ be the braid group on $n$-strings and let $\sigma_{1}, \ldots, \sigma_{n-1}$ denote the standard Artin generators. We are interested in closures of braids in $\mathbf{S}^{3}$. In general, the closure of a 
braid has many components. Here we describe the procedure which produces a knot from a braid. The closure of a braid $\alpha$ will be denoted either by $\operatorname{closure}(\alpha)$ or by $\widehat{\alpha}$.

Let us introduce some notation. Let ( ): $\mathbf{B}_{n} \rightarrow \Sigma_{n}$ be the projection onto the symmetric group on $n$ letters. The elements $\left(\sigma_{i}\right)$ are then the transpositions $(i, i+1)$. Let $\iota_{n}: \mathbf{B}_{n} \rightarrow \mathbf{B}_{n+1}$ denote the inclusion onto the first $n$ strands.

Let $\mathbf{B}_{n}^{K}$ denote the set of braids on $n$ strands consisting of braids whose closures are knots. It is a conjugation invariant set and it is the preimage of the set of the longest cycles with respect to the projection to the symmetric group. We define a projection $\pi_{n}: \mathbf{B}_{n} \rightarrow \mathbf{B}_{n}^{K}$ as follows.

Given a braid $\alpha \in \mathbf{B}_{n}$ we construct a braid $\sigma_{(\alpha)}$ depending only on the permutation $(\alpha) \in \Sigma_{n}$ induced by $\alpha$ such that the composed braid $\alpha \sigma_{(\alpha)}$ induces a longest cycle. More precisely, let

$$
(\alpha)=\left(a_{1,1} \cdots a_{1, n_{1}}\right)\left(a_{2,1} \cdots a_{2, n_{2}}\right) \cdots\left(a_{k, 1} \cdots a_{k, n_{k}}\right)
$$

be presented as a product of cycles such that $a_{1,1}<a_{2,1}<\cdots<a_{k, 1}$ and $a_{i, 1}<a_{i, j}$ for all $j=1, \ldots, n_{i}$. We also require that $\sum_{i=1}^{k} n_{i}=n$, that is, we list cycles of length one. Then we define

$$
\sigma_{(\alpha)}:=\sigma_{a_{2,1}-1} \sigma_{a_{3,1}-1} \cdots \sigma_{a_{k, 1}-1} .
$$

The permutation induced by $\alpha \sigma_{(\alpha)}$ is then the longest cycle obtained inductively by inserting the second cycle to the first one, the third cycle into the resulting cycle and so on.

A geometric description of the procedure goes as follows. Consider the closure of $\alpha$ and color the component containing the first strand red. Move to the left and if the $i^{\text {th }}$ strand is not red then multiply $\alpha$ by $\sigma_{i-1}$, extend the coloring and continue the procedure. The following properties are clear directly from the construction:

- The closure of $\alpha \sigma_{(\alpha)}$ is a knot.

- The braid $\sigma_{(\alpha)}$ is a product of $C_{\alpha}-1$ transpositions, where $C_{\alpha}$ is the number of components of the closure of $\alpha$.

- The closure of $\sigma_{(\alpha)}$ is a trivial link.

- If $\alpha \in \mathbf{B}_{n}^{K}$ then $\sigma_{(\alpha)}$ is the identity; in other words $\alpha \mapsto \alpha \sigma_{(\alpha)}$ defines a projection $\pi_{n}: \mathbf{B}_{n} \rightarrow \mathbf{B}_{n}^{K}$.

Next we define a map $\Psi_{n}: \mathbf{B}_{n} \rightarrow \operatorname{Conc}\left(\mathbf{S}^{3}\right)$ to be the composition of the projection $\pi_{n}$ followed by the closure of a braid and taking the concordance class:

$$
\Psi_{n}(\alpha)=\left[\operatorname{closure}\left(\pi_{n}(\alpha)\right)\right]=\left[\widehat{\alpha \sigma_{(\alpha)}}\right] .
$$


Now the proof of the following observation is straightforward.

Proposition 2.6 Let $\iota_{n}: \mathbf{B}_{n} \rightarrow \mathbf{B}_{n+1}$ be the inclusion onto the first $n$ strings. The following diagram is commutative:

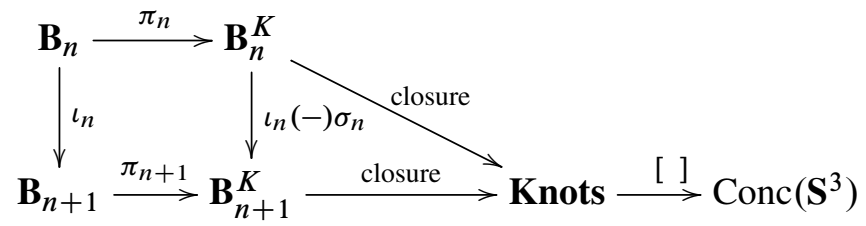

Consequently, $\Psi_{n+1} \circ \iota_{n}=\Psi_{n}$ and the map $\Psi_{\infty}: \mathbf{B}_{\infty} \rightarrow \operatorname{Conc}\left(\mathbf{S}^{3}\right)$ is well defined and surjective.

Remark The restriction of the map $\Psi_{\infty}$ to the commutator subgroup $\left[\mathbf{B}_{\infty}, \mathbf{B}_{\infty}\right]$ is surjective. To see this let $K=\Psi_{n}(\alpha)$ where $\alpha \in \mathbf{B}_{n}^{K}$. Suppose that $\operatorname{Ab}(\alpha)=k$, where $\mathrm{Ab}: \mathbf{B}_{n} \rightarrow \mathbf{Z}$ is the abelianization homomorphism. Observe that the closure of the braid $\iota(\alpha) \sigma_{n}^{-1} \cdots \sigma_{n+k-1}^{-1}$ is equal to $K$ and that this braid belongs to the commutator subgroup $\left[\mathbf{B}_{n+k}, \mathbf{B}_{n+k}\right]$.

\section{Proofs}

\subsection{General facts about quasihomomorphisms}

The following lemma will be used in the proof of Corollary 1.4. For $\alpha, \beta \in G$ the following notation is used: $\alpha^{\beta}=\beta \alpha \beta^{-1}$.

Lemma 3.2 Let $A$ be an abelian group equipped with a pseudonorm $v$ and let $\psi: G \rightarrow$ $A$ be a quasihomomorphism.

(1) The following inequalities hold for every $\alpha, \beta \in G$ :

- $v\left(\psi(\beta)+\psi\left(\beta^{-1}\right)\right) \leq v\left(\psi\left(1_{G}\right)\right)+D_{\psi}$.

- $v\left(\psi\left(\alpha^{\beta}\right)-\psi(\alpha)\right) \leq 2 D_{\psi}$.

- $v(\psi([\alpha, \beta])) \leq 3 D_{\psi}$.

(2) If $\psi$ is bounded on a set $S$ normally generating $G$, then it is Lipschitz with respect to the biinvariant word metric on $G$. In particular, $\psi$ is Lipschitz if $G$ is normally finitely generated.

(3) The restriction of $\psi$ to the commutator subgroup $[G, G]$ is Lipschitz with respect to the commutator length. The Lipschitz constant is bounded by $4 D_{\psi}$. 
Proof (1) All inequalities follow directly from the quasihomomorphism property:

- $v\left(\psi(\beta)+\psi\left(\beta^{-1}\right)\right)=v\left(\psi\left(1_{G}\right)+\psi(\beta)-\psi\left(1_{G}\right)+\psi\left(\beta^{-1}\right)\right) \leq v\left(\psi\left(1_{G}\right)\right)+D_{\psi}$.

- $v\left(\psi\left(\alpha^{\beta}\right)-\psi(\alpha)\right)$

$$
\begin{aligned}
& =v\left(\psi\left(\beta \alpha \beta^{-1}\right)+\psi\left(\beta^{-1}\right)-\psi\left(\alpha \beta^{-1}\right)+\psi\left(\alpha \beta^{-1}\right)-\psi\left(\beta^{-1}\right)-\psi(\alpha)\right) \\
& \leq v\left(\psi\left(\beta \alpha \beta^{-1}\right)+\psi\left(\beta^{-1}\right)-\psi\left(\alpha \beta^{-1}\right)\right)+v\left(\psi\left(\alpha \beta^{-1}\right)-\psi\left(\beta^{-1}\right)-\psi(\alpha)\right) \\
& \leq 2 D_{\psi} .
\end{aligned}
$$

- Since $v\left(\psi\left(\alpha \beta \alpha^{-1} \beta^{-1}\right)+\psi\left(\beta \alpha \beta^{-1}\right)-\psi(\alpha)\right) \leq D_{\psi}$, we get the following inequalities where the last one follows from the previous item.

$$
\begin{aligned}
v(\psi[\alpha, \beta])-v\left(\psi\left(\beta \alpha \beta^{-1}\right)-\psi(\alpha)\right) & \leq D_{\psi}, \\
v(\psi[\alpha, \beta]) & \leq D_{\psi}+v\left(\psi\left(\beta \alpha \beta^{-1}\right)-\psi(\alpha)\right), \\
v(\psi[\alpha, \beta]) & \leq 3 D_{\psi} .
\end{aligned}
$$

(2) Let $\alpha=s_{1}^{\beta_{1}} \ldots s_{k}^{\beta_{k}}$, where $s_{i} \in S$. According to the hypothesis $\psi$ is bounded on $S$. It follows from the previous part that $\psi$ is bounded, say by $C \geq 0$, on the set $S_{\text {conj }}$ of all conjugates of the elements of $S$. We have

$$
\begin{aligned}
v(\psi(\alpha))=v\left(\psi\left(s_{1}^{\beta_{1}} \ldots s_{k}^{\beta_{k}}\right)\right) & \leq v\left(\sum_{i=1}^{k} \psi\left(s_{i}^{\beta_{i}}\right)\right)+(k-1) D_{\psi} \\
& \leq \sum_{i=1}^{k} v\left(\psi\left(s_{i}^{\beta_{i}}\right)\right)+(k-1) D_{\psi} \leq\left(C+D_{\psi}\right) k,
\end{aligned}
$$

and the statement follows.

(3) The last inequality of item (1) shows that $\psi$ is bounded on commutators. This implies that if $\gamma \in[G, G]$ is a product of $k$ commutators then

$$
\begin{aligned}
v(\psi(\gamma))=v\left(\psi\left(\prod_{i=1}^{k}\left[\alpha_{i}, \beta_{i}\right]\right)\right) & \leq \sum_{i=1}^{k} v\left(\psi\left[\alpha_{i}, \beta_{i}\right]\right)+(k-1) D_{\psi} \\
& \leq k 3 D_{\psi}+(k-1) D_{\psi}=(4 k-1) D_{\psi} \leq 4 D_{\psi} k .
\end{aligned}
$$

Thus the Lipschitz constant of the restriction of $\psi$ to the commutator subgroup with respect to the commutator length is bounded by $4 D_{\psi}$.

\subsection{Proof of the first results and basic consequences}

Recall that given two knots $K$ and $K^{\prime}$ we denoted by $-K$ the knot $K$ with the reversed orientation, by $K^{*}$ the knot which is the mirror image of the knot $K$, and by $K \# K^{\prime}$ the 
connected sum of $K$ and $K^{\prime}$. In [1, Lemma 2.7], the first author proved the following lemma (we reproduce the proof for completeness).

Lemma 3.4 Let $\alpha, \beta \in \mathbf{B}_{n}$. There exists a smooth bordism $\Sigma \rightarrow \mathbf{B}^{4}$ between the knots

$$
\widehat{\alpha \sigma_{(\alpha)}} \# \widehat{\beta \sigma_{(\beta)}} \#-\left(\widehat{\alpha \beta \sigma_{(\alpha \beta)}}\right)^{*} \quad \text { and } \quad \widehat{\alpha \beta \sigma_{(\alpha \beta)}} \#-\left(\widehat{\alpha \beta \sigma_{(\alpha \beta)}}\right)^{*}
$$

such that $\chi(\Sigma) \geq-6 n$.

Proof The proof relies on the observation that if a link $L$ is obtained from a link $L^{\prime}$ by the operation presented in Figure 3.1 then there is an oriented bordism between $L$ and $L^{\prime}$ of Euler characteristic equal to -1 .

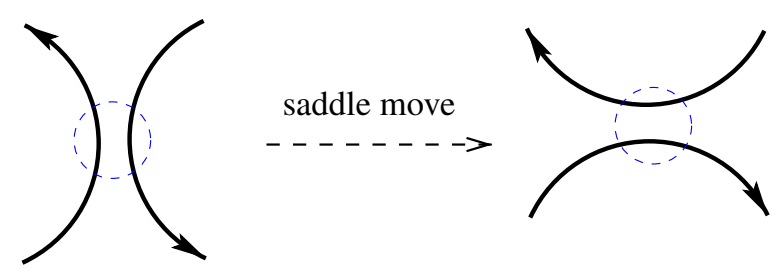

Figure 3.1: Saddle move which results in a cobordism of Euler characteristic -1 between links $L$ and $L^{\prime}$

Applying this argument inductively we get that the there is a bordism between $\widehat{\alpha \beta}$ and $\widehat{\alpha} \sqcup \widehat{\beta}$, where $\alpha, \beta \in \mathbf{B}_{n}$ and that the Euler characteristic of this bordism is equal to $-n$. In our situation we obtain the following sequence of bordisms (the number over an arrow is an upper bound on the number of one-handles attached to the previous bordism):

$$
\begin{aligned}
\widehat{\alpha \beta \sigma_{(\alpha \beta)}} \stackrel{2 n}{\longrightarrow} \widehat{\alpha} \sqcup \widehat{\beta} \sqcup \widehat{\sigma_{(\alpha \beta)}} \stackrel{2 n-1}{\longrightarrow} \widehat{\alpha} \sqcup \widehat{\beta} \sqcup \widehat{\sigma_{(\alpha)}} \sqcup \widehat{\sigma_{(\beta)}} \\
\stackrel{2 n}{\longrightarrow} \widehat{\alpha \sigma_{(\alpha)}} \sqcup \widehat{\beta \sigma_{(\beta)}} \stackrel{1}{\longrightarrow} \widehat{\alpha \sigma_{(\alpha)}} \# \widehat{\beta \sigma_{(\beta)}} .
\end{aligned}
$$

The number of handles in the second bordism follows from an observation that the closure $\widehat{\sigma_{(\gamma)}}$ is a trivial link with at most $n$ components for any $\gamma \in \mathbf{B}_{n}$. It follows that there is a bordism

$$
\widehat{\alpha \beta \sigma_{(\alpha \beta)}} \#-\left(\widehat{\alpha \beta \sigma_{(\alpha \beta)}}\right)^{*} \stackrel{6 n}{\longrightarrow} \widehat{\alpha \sigma_{(\alpha)}} \# \widehat{\beta \sigma_{(\beta)}} \#-\left(\widehat{\alpha \beta \sigma_{(\alpha \beta)}}\right)^{*},
$$

which is the cylinder with at most $6 n$ handles attached, which implies the statement.

Since the second knot in the above lemma is slice, we obtain that the four ball genus of the first knot is bounded by $3 n+1$. 
Proof of Theorem 1.1 Let $\alpha, \beta \in \mathbf{B}_{n}$. Then

$$
\mathrm{g}_{4}\left(\Psi_{n}(\alpha)+\Psi_{n}(\beta)-\Psi_{n}(\alpha \beta)\right)=\mathrm{g}_{4}\left[\widehat{\alpha \sigma_{(\alpha)}} \# \widehat{\beta \sigma_{(\beta)}} \#-\left(\widehat{\alpha \beta \sigma_{(\alpha \beta)}}\right)^{*}\right] \leq 3 n+1 .
$$

This proves that $\Psi_{n}: \mathbf{B}_{n} \rightarrow \operatorname{Conc}\left(\mathbf{S}^{3}\right)$ is a quasihomomorphism with defect bounded by $3 n+1$.

Let us specify the general inequalities from Lemma 3.2 to our situation.

Corollary 3.5 The quasihomomorphism $\Psi_{n}: \mathbf{B}_{n} \rightarrow \operatorname{Conc}\left(\mathbf{S}^{3}\right)$ satisfies the following inequalities for every $\alpha, \beta \in \mathbf{B}_{n}$ :

- $\mathrm{g}_{4}\left(\Psi_{n}(\alpha)+\Psi_{n}\left(\alpha^{-1}\right) \leq D_{\Psi_{n}} \leq 3 n+1\right.$.

- $\mathrm{g}_{4}\left(\Psi_{n}\left(\alpha^{\beta}-\Psi_{n}(\alpha)\right) \leq 2 D_{\Psi_{n}} \leq 6 n+2\right.$.

- $\mathrm{g}_{4}\left(\Psi_{n}([\alpha, \beta])\right) \leq 3 D_{\Psi_{n}} \leq 9 n+3$.

Proof Since the closure of $\sigma_{1} \cdots \sigma_{n-1}$ is the unknot we get that $\Psi_{n}\left(1_{\mathbf{B}_{n}}\right)$ is equal to the trivial concordance class and hence $\mathrm{g}_{4}\left(\Psi_{n}\left(1_{\mathbf{B}_{n}}\right)\right)=0$. Consequently, the above inequalities follow directly from Lemma 3.2 and Theorem 1.1.

Proposition 3.6 The sequence of the defects of the quasihomomorphisms $\Psi_{n}: \mathbf{B}_{n} \rightarrow$ $\operatorname{Conc}\left(\mathbf{S}^{3}\right)$ is unbounded:

$$
\limsup _{n \rightarrow \infty} D_{\Psi_{n}}=\infty
$$

Proof If the defects were uniformly bounded then the map $\Psi_{\infty}$ would be a quasihomomorphism. This would imply, according to Kotschick [8], that the composition $\mathbf{B}_{3} \rightarrow \mathbf{B}_{\infty} \rightarrow \operatorname{Conc}\left(\mathbf{S}^{3}\right) \rightarrow \mathbf{R}$, where the last map is given by the signature link invariant, is a bounded distance from a homomorphism. However, it is known that this composition is a quasimorphism, whose homogenization which does not vanish on the commutator subgroup $\left[\mathbf{B}_{3}, \mathbf{B}_{3}\right]$. This follows, for example, from the fact that the nontrivial homogeneous signature quasimorphism $\overline{\operatorname{sign}}_{3}$ on $\mathbf{B}_{3}$ defined in [5] is not a homomorphism. Indeed, if it is a nontrivial homomorphism, then its value on the braid $\eta_{3,3}$ must be equal to twice its value on the braid $\eta_{2,3}$, where the braids $\eta_{2,3}$ and $\eta_{3,3}$ are shown in Figure 4.1. However, in [5] Gambaudo and Ghys showed that $\overline{\operatorname{sign}}_{3}\left(\eta_{2,3}\right)=\overline{\operatorname{sign}}_{3}\left(\eta_{3,3}\right)=2$.

Remark The above proposition does not exclude the possibility that $\Psi_{\infty}: \mathbf{B}_{\infty} \rightarrow$ $\operatorname{Conc}\left(\mathbf{S}^{3}\right)$ is Lipschitz. It can't be Lipschitz, however, with respect to the commutator length because the latter is bounded by two on the infinite braid group [3, Theorem 2.2]. 


\section{Proof of Theorem 1.2}

The main ingredient of the proof is the following observation.

Lemma 3.7 Let $\alpha, \beta \in \mathbf{B}_{n}$ be braids. Suppose that

$$
\alpha=\sigma_{i_{1}}^{ \pm 1} \cdots \sigma_{i_{m}}^{ \pm 1} \in \mathbf{B}_{n} \quad \text { and } \quad \beta=\sigma_{i_{1}}^{ \pm 1} \cdots \sigma_{i_{k-1}}^{ \pm 1} \sigma_{i_{k+1}}^{ \pm 1} \cdots \sigma_{i_{m}}^{ \pm 1} .
$$

That is, $\beta$ is obtained from $\alpha$ by removing one crossing. Then there is a smooth bordism $\Sigma \rightarrow \mathbf{B}^{4}$ from the closure $\hat{\alpha}$ to the closure $\widehat{\beta}$ whose Euler characteristic is equal to -1 .

Proof It is enough to argue locally at a neighborhood of a crossing. The proof for removal of $\sigma_{i}$ is presented in Figure 3.2. The proof for removal of $\sigma_{i}^{-1}$ is analogous.

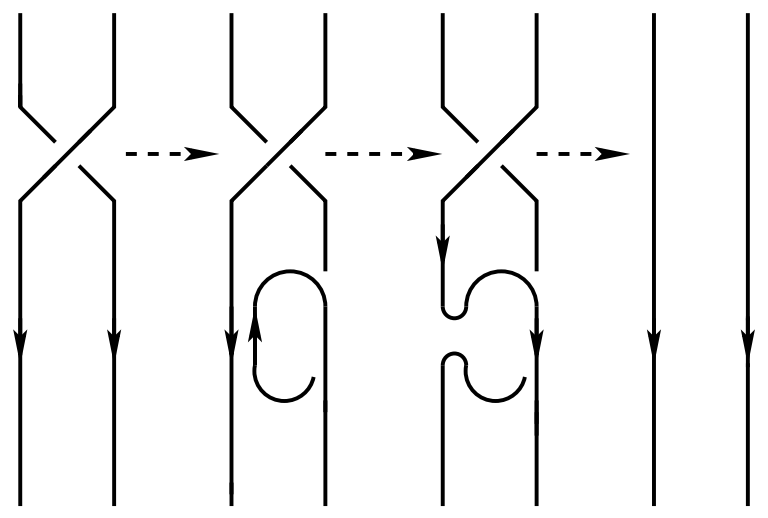

Figure 3.2: Local moves

The first step is to change the braid after the crossing by the first Reidemeister move (appropriately chosen) so the neighboring strings go in opposite directions. Next we perform a saddle move. Then we apply the second Reidemeister move which results in the braid $\beta$. We obtain that there is a bordism from the closure of $\alpha$ to the closure of $\beta$ whose Euler characteristic is equal to -1 .

Proof of Theorem 1.2 Let us first recall that all generators $\sigma_{i} \in \mathbf{B}_{n}$ are conjugate to each other and hence $\mathbf{B}_{n}$ is normally generated by $\sigma_{1}$ and its inverse. Let $\alpha \in \mathbf{B}_{n}$. Suppose that $\|\alpha\|=k$ which means that

$$
\alpha=\beta_{1} \sigma_{1}^{ \pm 1} \beta_{1}^{-1} \cdots \beta_{k} \sigma_{1}^{ \pm 1} \beta_{k}^{-1}
$$

for some $\beta_{i} \in \mathbf{B}_{n}$. The knot $\Psi_{n}(\alpha)$ is the closure of the braid

$$
\beta_{1} \sigma_{1}^{ \pm 1} \beta_{1}^{-1} \cdots \beta_{k} \sigma_{1}^{ \pm 1} \beta_{k}^{-1} \sigma_{(\alpha)} .
$$


By applying Lemma $3.7 k$ times we remove the crossings corresponding to $\sigma_{1}^{ \pm 1}$ in the above presentation and we obtain that the above closure is bordant to the closure of $\sigma_{(\alpha)}$ via a bordism whose Euler characteristic is equal to $-k$.

The closure of $\sigma_{(\alpha)}$ is a trivial link and we cap off all its components. This increases the Euler characteristic by the number of components which is at least one and yields a surface of genus at most $k / 2$ in $\mathbf{B}^{4}$ bounded by $\Psi_{n}(\alpha)$. We get that

$$
\mathrm{g}_{4}\left(\Psi_{n}(\alpha)\right) \leq \frac{k}{2}=\frac{1}{2}\|\alpha\|
$$

as claimed.

\subsection{Proofs of the corollaries}

Proof of Corollary 1.3 Let $\alpha, \beta \in \mathbf{B}_{n}$ and let $\varphi$ : $\operatorname{Conc}\left(\mathbf{S}^{3}\right) \rightarrow \mathbf{R}$ be a quasimorphism which is Lipschitz with respect to the four ball genus norm. Let $C_{\varphi}$ and $D_{\varphi}$ denote its Lipschitz constant and the defect respectively. Recall that the defect of $\Psi_{n}$ satisfies $D_{\Psi_{n}} \leq 3 n+1$, due to Theorem 1.1.

Recall that for any $\alpha, \beta \in \mathbf{B}_{n}$ the quasimorphism property of $\Psi_{n}$ says that

$$
g_{4}\left(\Psi_{n}(\alpha)-\Psi_{n}(\alpha \beta)+\Psi_{n}(\beta)\right) \leq D_{\Psi_{n}}
$$

and then it is a consequence of the Lipschitz property of $\varphi$ that

$$
\left|\varphi\left(\Psi_{n}(\alpha)-\Psi_{n}(\alpha \beta)+\Psi_{n}(\beta)\right)\right| \leq C_{\varphi} D_{\Psi_{n}} .
$$

The defect of the composition $\varphi \circ \Psi_{n}$ is estimated as

$$
\begin{aligned}
\mid \varphi\left(\Psi_{n}(\alpha)\right) & -\varphi\left(\Psi_{n}(\alpha \beta)\right)+\varphi\left(\Psi_{n}(\beta)\right) \mid \\
& \leq\left|\varphi\left(\Psi_{n}(\alpha)\right)+\varphi\left(-\Psi_{n}(\alpha \beta)\right)+\varphi\left(\Psi_{n}(\beta)\right)\right|+\left|\varphi\left(\Psi_{n}(\alpha \beta)\right)+\varphi\left(-\Psi_{n}(\alpha \beta)\right)\right| \\
& \leq\left|\varphi\left(\Psi_{n}(\alpha)-\Psi_{n}(\alpha \beta)+\Psi_{n}(\beta)\right)\right|+3 D_{\varphi}+\left|\varphi\left(1_{\operatorname{Conc}\left(\mathbf{S}^{3}\right)}\right)\right| \\
& \leq C_{\varphi} D_{\Psi_{n}}+3 D_{\varphi}+\left|\varphi\left(1_{\operatorname{Conc}\left(\mathbf{S}^{3}\right)}\right)\right| \\
& \leq(3 n+1) C_{\varphi}+3 D_{\varphi}+\left|\varphi\left(1_{\operatorname{Conc}\left(\mathbf{S}^{3}\right)}\right)\right|,
\end{aligned}
$$

where the second inequality follows from the quasimorphism property of $\varphi$ and Lemma 3.2.

Proof of Corollary 1.4 First we prove that the quasihomomorphism $\Psi_{n}$ is Lipschitz with respect to the biinvariant word metric. Let $\alpha \in \mathbf{B}_{n}$ be an element of the biinvariant 
word norm $\|\alpha\|$ equal to $k$. This means that $\alpha=\left(\sigma_{1}^{ \pm 1}\right)^{p_{1}} \ldots\left(\sigma_{1}^{ \pm}\right)^{p_{k}}$ for some $p_{i} \in \mathbf{B}_{n}$. Then

$$
\begin{aligned}
\mathrm{g}_{4}\left(\Psi_{n}(\alpha)\right)=\mathrm{g}_{4}\left(\Psi_{n}\left(\sigma_{1}^{ \pm 1}\right)^{p_{1}} \cdots\left(\sigma_{1}^{ \pm}\right)^{p_{k}}\right) & \leq \sum_{i=1}^{k} \mathrm{~g}_{4}\left(\Psi_{n}\left(\left(\sigma_{1}^{ \pm 1}\right)^{p_{i}}\right)\right)+(k-1) D_{\Psi_{n}} \\
& \leq D_{\Psi_{n}}(k-1) \leq D_{\Psi_{n}}\|\alpha\| \leq(3 n+1)\|\alpha\| .
\end{aligned}
$$

The first inequality follows from the quasihomomorphism property and the second inequality is a consequence of the fact that the closure of $\sigma_{1}^{ \pm 1}$ is the unknot.

Let us now consider the restriction of $\Psi_{n}$ to the commutator subgroup $\left[\mathbf{B}_{n}, \mathbf{B}_{n}\right]$ equipped with the commutator length. Since

$$
\mathrm{g}_{4}\left(\Psi_{n}[\alpha, \beta]\right) \leq 3 D_{\Psi_{n}} \leq 9 n+3,
$$

due to Corollary 3.5, we get that if $\alpha \in\left[\mathbf{B}_{n}, \mathbf{B}_{n}\right]$, then

$$
\mathrm{g}_{4}\left(\Psi_{n}(\alpha)\right) \leq 4 D_{\Psi_{n}} \operatorname{cl}(\alpha) \leq(12 n+4) \operatorname{cl}(\alpha) .
$$

Proof of Corollary 1.5 Let $\alpha \in\left[\mathbf{B}_{n}, \mathbf{B}_{n}\right]$. It follows from the quasihomomorphism property that

$$
\mathrm{g}_{4}\left(\Psi_{n}\left(\alpha^{k}\right)-k \Psi_{n}(\alpha)\right) \leq(k-1) D_{\Psi_{n}} .
$$

By dividing by $k$ and taking the limit we obtain that

$$
\left|\limsup _{k \rightarrow \infty} \frac{g_{4}\left(\Psi_{n}\left(\alpha^{k}\right)\right)}{k}-\operatorname{sg}_{4}\left(\Psi_{n}(\alpha)\right)\right| \leq D_{\Psi_{n}},
$$

Since $\Psi_{n}$ is Lipschitz with respect to the commutator length (the Lipschitz constant is computed in the proof of Corollary 1.4), we have that

$$
\limsup _{k \rightarrow \infty} \frac{\mathrm{g}_{4}\left(\Psi_{n}\left(\alpha^{k}\right)\right)}{k} \leq \lim _{k \rightarrow \infty} \frac{4 D_{\Psi_{n}} \operatorname{cl}\left(\alpha^{k}\right)}{k}=4 D_{\Psi_{n}} \operatorname{scl}(\alpha) .
$$

It then follows from Equation (3-1) that

$$
\operatorname{sg}_{4}\left(\Psi_{n}(\alpha)\right) \leq 4 D_{\Psi_{n}} \operatorname{scl}(\alpha)+D_{\Psi_{n}}
$$

as claimed.

Proof of Corollary 1.6 Since the map $\Psi_{n}$ is Lipschitz by Corollary 1.4 we obtain that

$$
\mathrm{g}_{4}\left(\Psi_{n}\left(\alpha^{k}\right)\right) \leq(3 n+1)\left\|\alpha^{k}\right\| .
$$


The stable commutator length of $\alpha$ is trivial which implies, according to the bqdichotomy (see Section 2.3), that the cyclic subgroup generated by $\alpha$ is bounded. As a consequence we get the uniform bound on the four ball genus of $\Psi_{n}\left(\alpha^{k}\right)$.

Proof of Corollary 1.9 Recall that $\varphi$ : $\operatorname{Conc}\left(\mathbf{S}^{3}\right) \rightarrow \mathbf{R}$ is a Lipschitz quasimorphism with the Lipschitz constant $C_{\varphi}$. Let $\alpha \in \mathbf{B}_{n}$ and $p \in \mathbf{N}$. Then

$$
\begin{aligned}
\operatorname{sg}_{4}\left(\Psi_{n}\left(\alpha^{p}\right)\right)=\limsup _{k} \frac{g_{4}\left(k \Psi_{n}\left(\alpha^{p}\right)\right)}{k} & \geq \limsup _{k} \frac{g_{4}\left(\Psi_{n}\left(\alpha^{k p}\right)\right)-(k-1) D_{\Psi_{n}}}{k} \\
& \geq \frac{1}{C_{\varphi}} \limsup _{k} \frac{\left|\left(\varphi \circ \Psi_{n}\right)\left(\alpha^{k p}\right)\right|}{k}-D_{\Psi_{n}} \\
& \left.\geq \frac{1}{C_{\varphi}} \mid \overline{\left(\varphi \circ \Psi_{n}\right.}\right)\left(\alpha^{p}\right) \mid-D_{\Psi_{n}} \\
& =\frac{\left|\left(\overline{\varphi \circ \Psi_{n}}\right)(\alpha)\right|}{C_{\varphi}} \cdot p-D_{\Psi_{n}} .
\end{aligned}
$$

The first inequality follows from the quasihomomorphism property, the second is the Lipschitz property of $\varphi$ and the third is the definition of the homogenization of a quasimorphism.

Proof of Corollary 1.12 Let $\alpha \in \mathbf{B}_{n}$ and assume that $\alpha \in\left[\mathbf{B}_{\infty}, \mathbf{B}_{\infty}\right]$. Since the abelianizations homomorphisms $\mathbf{B}_{n} \rightarrow \mathbf{Z}$ commute with inclusions $\mathbf{B}_{n} \rightarrow \mathbf{B}_{n+1}$, we have that $\alpha \in\left[\mathbf{B}_{n}, \mathbf{B}_{n}\right]$. Let $\varphi: \operatorname{Conc}\left(\mathbf{S}^{3}\right) \rightarrow \mathbf{R}$ be a Lipschitz quasimorphism such that $\varphi\left(\Psi_{n}(\alpha)\right) \neq 0$. We have the inequalities

$$
\frac{\left|\overline{\left(\varphi \circ \Psi_{n}\right.}(\alpha)\right|}{C_{\varphi}} \cdot p-D_{\Psi_{n}} \leq \operatorname{sg}_{4}\left(\Psi_{n}\left(\alpha^{p}\right)\right) \leq \mathrm{g}_{4}\left(\Psi_{n}\left(\alpha^{p}\right)\right)=\mathrm{g}_{4}\left(\Psi_{\infty}\left(\alpha^{p}\right)\right) \leq \frac{1}{2}\left\|\alpha^{p}\right\| .
$$

The first inequality follows from Corollary 1.9 , the second one is obvious, the equality follows from Proposition 2.6 and the last one follows from Theorem 1.2. By dividing by $p$ and passing to the limit with $p \rightarrow \infty$ we obtain that $\lim _{p \rightarrow \infty}\left\|\alpha^{p}\right\| / p>0$ as claimed.

\subsection{Strong displaceability of braids}

Let $m \in \mathbf{N}$ be a natural number. A subgroup $H \subset G$ is called strongly $m$-displaceable if there exists $\mathrm{g} \in \mathrm{G}$ such that the conjugate subgroups $\mathrm{g}^{i} \mathrm{Hg}^{-i}$ and $\mathrm{g}^{j} \mathrm{Hg}^{-j}$ commute for $0 \leq i<j \leq m$.

Proposition 3.10 For every natural numbers $m, n \in \mathbf{N}$, the braid group $\mathbf{B}_{n}$ is strongly $m$-displaceable in $\mathbf{B}_{\infty}$ and the commutator subgroup $\left[\mathbf{B}_{n}, \mathbf{B}_{n}\right]$ is strongly $m$-displaceable in $\left[\mathbf{B}_{\infty}, \mathbf{B}_{\infty}\right]$. 
Proof For every $n \in \mathbf{N}$ we define an argyle braid $A_{n, i}$ to be

$$
A_{n, i}:=\prod_{k=1}^{n} \prod_{j=1}^{n} \sigma_{i n-k+j} .
$$

This is a braid that swaps the $i^{\text {th }} n$ strings with the $(i+1)^{\text {st }} n$ strings, it is a product of $n^{2}$ standard generators and it looks like an argyle pattern. For example, $A_{1, i}=\sigma_{i}$. If $n$ is even then by making the pattern alternating we define a commutator argyle braid by

$$
A_{n, i}^{\prime}:=\prod_{k=1}^{n} \prod_{j=1}^{n} \sigma_{i n-k+j}^{(-1)^{j}} .
$$

An example of $A_{4,1}^{\prime}$ drawn in Figure 3.3.

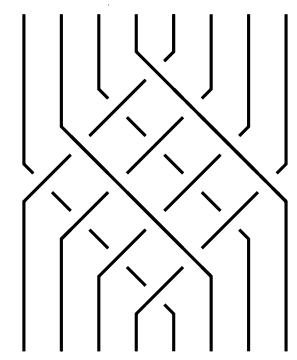

Figure 3.3: The commutator argyle braid $A_{4,1}^{\prime}$

Let $\mathbf{B}_{n} \subset \mathbf{B}_{\infty}$, where $n$ is a positive and even natural number. For any $m \in \mathbf{N}$ we define

$$
\Delta:=A_{n, 1}^{\prime} A_{n, 2}^{\prime} \cdots A_{n, m-1}^{\prime} \in\left[\mathbf{B}_{m n}, \mathbf{B}_{m n}\right] .
$$

The braid $\Delta$ is presented in Figure 3.4 in which each line represents $n$ strings and each crossing is the appropriate argyle braid $A_{n, i}^{\prime}$.

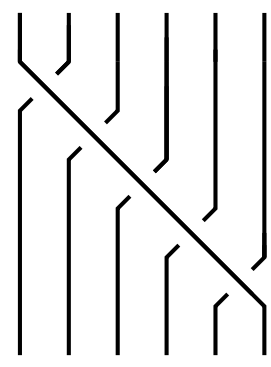

Figure 3.4: The braid $\Delta$ for $m=6$ 
It shows that the group $\mathbf{B}_{n}$ is strongly $m$-displaceable in $\mathbf{B}_{m n}$ by the braid $\Delta$ and that the commutator subgroup $\left[\mathbf{B}_{n}, \mathbf{B}_{n}\right]$ is strongly $m$-displaceable in $\left[\mathbf{B}_{m n}, \mathbf{B}_{m n}\right]$ which finishes the proof.

\section{Examples}

\subsection{Bounded cyclic subgroup of braid groups}

The following lemma was proved in [2] but, since its proof is short, we present it for completeness.

Lemma 4.2 Let $G$ be a normally finitely generated group and let $\alpha, \Delta \in G$. If $\alpha$ commutes with the conjugate $\alpha^{\Delta}$ then the cyclic subgroup generated by $[\alpha, \Delta]$ is bounded with respect to the biinvariant word metric on $G$. In particular, if $\alpha$ is conjugate to its inverse then $\alpha$ generates a bounded cyclic subgroup.

Proof An induction argument yields the equality

$$
[\alpha, \Delta]^{n}=\left[\alpha^{n}, \Delta\right]
$$

This implies that $\left\|[\alpha, \Delta]^{n}\right\|=\left\|\left[\alpha^{n}, \Delta\right]\right\| \leq 2\|\Delta\|$ which finishes the general part. If $\alpha$ is conjugate to its inverse via $\Delta$ then $\alpha^{2}=[\alpha, \Delta]$ and the second statement follows. Moreover, the braid $\left\|\alpha^{n}\right\|$ is bounded by $2\|\Delta\|+\|\alpha\|$ in this case.

Example 4.3 Let $\sigma_{1} \sigma_{2}^{-1} \in \mathbf{B}_{3}$ and let $\Delta=\sigma_{1} \sigma_{2} \sigma_{1}$ be the Garside element (the half twist). Observe that

$$
\left(\sigma_{1} \sigma_{2}^{-1}\right)^{\Delta}=\sigma_{2} \sigma_{1}^{-1},
$$

that is $\sigma_{1} \sigma_{2}^{-1}$ is conjugate to its inverse. It then follows from Lemma 4.2 that the braid $\sigma_{1} \sigma_{2}^{-1}$ generates a cyclic subgroup in $\mathbf{B}_{3}$ bounded by $8=2\|\Delta\|+\left\|\sigma_{1} \sigma_{2}^{-1}\right\|$.

Example 4.4 Let $\sigma_{1} \sigma_{3}^{-1} \in \mathbf{B}_{4}$. It is conjugate to its inverse via the braid which swaps the first two and the last two strings. More precisely, the conjugating braid is given in this case by $\Delta=\sigma_{2} \sigma_{1} \sigma_{3} \sigma_{2}$. Again, by the above lemma we obtain that $\sigma_{1} \sigma_{3}^{-1}$ generates a cyclic subgroup of $\mathbf{B}_{4}$ bounded by $10=2\|\Delta\|+\left\|\sigma_{1} \sigma_{3}^{-1}\right\|$. 


\subsection{The notorious family $\Psi_{3}\left(\left(\sigma_{1} \sigma_{2}^{-1}\right)^{n}\right)$}

We saw in the previous section that the cyclic subgroup generated by $\sigma_{1} \sigma_{2}^{-1}$ is bounded in $\mathbf{B}_{3}$. According to Corollary 1.4, Corollary 1.6 and Example 4.3 we get the following bound on the four ball genus

$$
\mathrm{g}_{4}\left(\Psi_{3}\left(\left(\sigma_{1} \sigma_{2}^{-1}\right)^{n}\right)\right) \leq \frac{1}{2}\left\|\left(\sigma_{1} \sigma_{2}^{-1}\right)^{n}\right\| \leq 4 .
$$

So we obtain a very simple example of a infinite family of knots with uniformly bounded four ball genus. However, this family has been notorious in the sense that it remains an open problem whether the induced family of concordance classes $\Psi_{3}\left(\left(\sigma_{1} \sigma_{2}^{-1}\right)^{n}\right)$ for $n$ not divisible by three is infinite. ${ }^{1}$ It is known that these concordance classes are of order at most two, since these knots are amphicheiral, so the question is whether these knots are slice. By the work of Long [11], if $n$ is odd and not divisible by 3 then the closure of the braid $\left(\sigma_{1} \sigma_{2}^{-1}\right)^{n}$ is strongly plus-amphicheiral and hence it is algebraically slice.

\subsection{The family $\Psi_{4}\left(\left(\sigma_{1} \sigma_{3}^{-1}\right)^{n}\right)$}

The same argument as above shows that the four ball genus of the knots $\Psi_{4}\left(\left(\sigma_{1} \sigma_{3}^{-1}\right)^{n}\right)$ is bounded by $\left.\frac{1}{2} \|\left(\sigma_{1} \sigma_{3}^{-1}\right)^{n}\right) \|=5$. In this case, however, we know that the set of induced concordance classes is infinite. More precisely, we have the following result.

Proposition 4.7 Let $\gamma=\sigma_{1}^{2} \sigma_{3}^{-2} \in \mathbf{B}_{4}$. Then the set $\left\{\Psi_{4}\left(\gamma^{n}\right)\right\}_{n=1}^{\infty}$ is infinite in $\operatorname{Conc}\left(\mathbf{S}^{3}\right)$, and the four ball genus of $\Psi_{4}\left(\gamma^{n}\right)$ is uniformly bounded. Moreover, there exists an increasing sequence of natural numbers $\left\{n_{i}\right\}_{i=1}^{\infty}$ such that the set $\left\{\Psi_{4}\left(\gamma^{n_{i}}\right)\right\}_{i=1}^{\infty}$ generates $\mathbf{Z}^{\infty}$ in $\operatorname{Conc}\left(\mathbf{S}^{3}\right)$.

Proof By definition $\Psi_{4}\left(\gamma^{n}\right)$ equals to the concordance class of the closure of the braid $\sigma_{1}^{2 n} \sigma_{3}^{-2 n} \sigma_{1} \sigma_{2} \sigma_{3}$. For $n \in \mathbf{N}$ denote by $T_{2 n-1}$ the knot obtained by taking a closure of the braid $\sigma_{1}^{2 n-1} \in \mathbf{B}_{2}$. It follows that the closure of the braid $\sigma_{1}^{2 n} \sigma_{3}^{-2 n} \sigma_{1} \sigma_{2} \sigma_{3}$ equals to the knot $T_{2 n+1} \#\left(T_{2 n-1}\right)^{*}$.

The knot $T_{2 n+1}$ is a $(2,2 n+1)$ torus knot. Hence

$$
\Delta_{T_{2 n+1}}(t)=\frac{\left(t^{4 n+2}-1\right)(t-1)}{\left(t^{2}-1\right)\left(t^{2 n+1}-1\right)}=\sum_{i=0}^{2 n}(-1)^{i} t^{2 n-i},
$$

\footnotetext{
${ }^{1}$ Paolo Lisca told the authors of this history.
} 
where $\Delta_{K}(t)$ is the Alexander polynomial of a knot $K$. It follows that

$$
\begin{gathered}
\operatorname{det}\left(T_{2 n+1}\right)=(2 n+1), \quad \operatorname{det}\left(\left(T_{2 n-1}\right)^{*}\right)=2 n-1, \\
\operatorname{det}\left(T_{2 n+1} \#\left(T_{2 n-1}\right)^{*}\right)=(2 n+1)(2 n-1),
\end{gathered}
$$

where the determinant of $K$ is defined to be $\operatorname{det}(K):=\left|\Delta_{K}(-1)\right|$. Let $\left\{n_{i}\right\}_{i=1}^{\infty}$ be an increasing sequence of natural numbers such that $p_{i}:=2 n_{i}+1$ is a prime number. It follows that for each $i \in \mathbf{N}$ we have

$$
\operatorname{det}\left(T_{p_{i}} \#\left(T_{p_{i}-2}\right)^{*}\right)=p_{i}\left(p_{i}-2\right) .
$$

Hence $\operatorname{det}\left(T_{p_{i}} \#\left(T_{p_{i}-2}\right)^{*}\right)$ is not a square, and for each $i>j \in \mathbf{N}$

$$
\operatorname{det}\left(T_{p_{i}} \#\left(T_{p_{i}-2}\right)^{*} \#-\left(T_{p_{j}}\right)^{*} \#-\left(T_{p_{j}-2}\right)\right)=p_{i}\left(p_{i}-2\right) p_{j}\left(p_{j}-2\right)
$$

is not a square. Since the determinant of a slice knot must be a square number, the concordance classes of knots $\left(T_{p_{i}} \#\left(T_{p_{i}-2}\right)\right)$ are pairwise distinct. Hence the set $\left\{\Psi_{4}\left(\gamma^{n_{i}}\right)\right\}_{i=1}^{\infty}$ is infinite in $\operatorname{Conc}\left(\mathbf{S}^{3}\right)$.

Let $\left\{p_{i}\right\}_{i=1}^{\infty}$ be a set of odd primes such that for each $i$

$$
p_{i}>2 p_{1} \cdots p_{i-1} .
$$

Let $n_{i}:=p_{1} \cdots p_{i}$. In what follows we are going to show that the set $\left\{\Psi_{4}\left(\gamma^{n_{i}}\right)\right\}_{i=1}^{\infty}$ generates $\mathbf{Z}^{\infty}$ in $\operatorname{Conc}\left(\mathbf{S}^{3}\right)$.

Recall that for each complex number $\omega \neq 1$, such that $|\omega|=1$, there exists the $\omega-$ signature (Levine-Tristram $\omega$-signature) homomorphism $\operatorname{sign}_{\omega}: \operatorname{Conc}\left(\mathbf{S}^{3}\right) \rightarrow \mathbf{Z}$. For each odd prime $p$ denote by $\omega_{p}:=\exp ((p-1) \pi i / p)$. It follows from [14, Lemma 3.5] that for each prime $p$ and each natural number $n$ we have

$$
\operatorname{sign}_{\omega_{p}}\left(\Psi_{4}\left(\gamma^{n}\right)\right)=\operatorname{sign}_{\omega_{p}}\left(T_{2 n+1} \#\left(T_{2 n-1}\right)^{*}\right)=2-2\left(\left[\frac{n}{p}+\frac{1}{2 p}\right]-\left[\frac{n}{p}-\frac{1}{2 p}\right]\right),
$$

where [ $\cdot]$ denotes the integer part. Since $p_{i}>2 p_{1} \cdots p_{i-1}$ and $n_{i}:=p_{1} \cdots p_{i}$ we obtain

$$
\begin{aligned}
& \operatorname{sign}_{\omega_{p_{i+1}}}\left(\Psi_{4}\left(\gamma^{n_{i}}\right)\right)=\operatorname{sign}_{\omega_{p_{i+1}}}\left(T_{2 n_{i}+1} \#\left(T_{2 n_{i}-1}\right)^{*}\right)=2, \\
& \operatorname{sign}_{\omega_{p_{i+1}}}\left(\Psi_{4}\left(\gamma^{n_{j}}\right)\right)=\operatorname{sign}_{\omega_{p_{i+1}}}\left(T_{2 n_{j}+1} \#\left(T_{2 n_{j}-1}\right)^{*}\right)=0 \quad \text { if } i+1<j,
\end{aligned}
$$

and the proof follows.

Remark Note that the standard 3-dimensional genus of knots $\Psi_{4}\left(\gamma^{n}\right)$ goes to infinity when $n \rightarrow \infty$, since the genus of $T_{2 n+1}=n$ and thus the genus of $\Psi_{4}\left(\gamma^{n}\right)$ equals to $2 n-1$. 


\subsection{Prime knots with unbounded stable genus}

This section provides details for Example 1.10. We construct a braid $\alpha \in \mathbf{B}_{n}$ for $n \geq 3$ such that the four ball genus of the knots $\Psi_{n}\left(\alpha^{p}\right)$ grows linearly with $p$.

Let $\operatorname{sign}(L) \in \mathbf{Z}$ denote the signature invariant of a link $L$. The restriction of the signature to knots descends to a homomorphism

$$
\text { sign: } \operatorname{Conc}\left(\mathbf{S}^{3}\right) \rightarrow \mathbf{Z}
$$

on the concordance group. It is a well-known fact due to Murasugi [13] that the inequality

$$
|\operatorname{sign}(K)| \leq 2 \mathrm{~g}_{4}(K)
$$

holds for every knot $K$. In other words the signature is Lipschitz with constant $C_{\text {sign }}=2$. It follows from Corollary 1.3 that the composition sign $\circ \Psi_{n}: \mathbf{B}_{n} \rightarrow \mathbf{R}$ is a quasimorphism on the braid group.

In order to apply Corollary 1.9 we need to show that the there exists a braid $\alpha \in \mathbf{B}_{n}$ such that the quasimorphism sign $\circ \Psi_{n}$ is unbounded on the cyclic subgroup generated by $\alpha$.

Let $\eta_{i, n}:=\sigma_{i-1} \cdots \sigma_{2} \sigma_{1}^{2} \sigma_{2} \cdots \sigma_{i-1} \in \mathbf{B}_{n}$, be the braid presented in Figure 4.1.

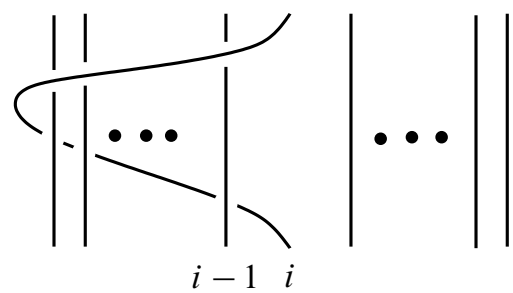

Figure 4.1: The braid $\eta_{i, n}$

Let $\alpha=\eta_{2, n}^{-2} \eta_{3, n} \in \mathbf{B}_{n}$. Observe that $\alpha=\sigma_{1}^{-4} \sigma_{2} \sigma_{1}^{2} \sigma_{2}$ and hence we get that $\alpha \in\left[\mathbf{B}_{n}, \mathbf{B}_{n}\right]$. Notice moreover that $\alpha$ is a pure braid and hence for each integer $p$ we have $\sigma_{\left(\alpha^{p}\right)}=\delta$, where $\delta:=\sigma_{1} \cdots \sigma_{n-1}$.

Let $\operatorname{sign}_{n}: \mathbf{B}_{n} \rightarrow \mathbf{Z}$ be a function defined by $\operatorname{sign}_{n}(\beta)=\operatorname{sign}(\widehat{\beta})$. Gambaudo and Ghys showed in [5] that $\operatorname{sign}_{n}$ is a quasimorphism on $B_{n}$ with a defect $D_{\operatorname{sign}_{n}} \leq n-1$. We denote by $\overline{\operatorname{sign}}_{n}$ the induced homogeneous quasimorphism. They also proved that

$$
\overline{\operatorname{sign}}_{n}\left(\eta_{i, n}\right)= \begin{cases}i & \text { if } i \text { is even, } \\ i-1 & \text { if } i \text { is odd. }\end{cases}
$$


Since the braids $\eta_{i, n}$ pairwise commute we have

$$
\overline{\operatorname{sign}}_{n}(\alpha)=\overline{\operatorname{sign}}_{n}\left(\eta_{2, n}^{-2}\right)+\overline{\operatorname{sign}}_{n}\left(\eta_{3, n}\right)=-2 .
$$

Since the closure $\widehat{\delta}$ is the unknot we get that

$$
\left|\operatorname{sign}_{n}\left(\alpha_{n}^{p} \delta\right)-\operatorname{sign}_{n}\left(\alpha_{n}^{p}\right)\right| \leq n-1,
$$

for every integer $p \in \mathbf{Z}$. It follows from a general fact about the homogenization of quasimorphisms that

$$
\left|\operatorname{sign}_{n}\left(\alpha_{n}^{p}\right)-\overline{\operatorname{sign}}_{n}\left(\alpha_{n}^{p}\right)\right| \leq D_{\operatorname{sign}_{n}} \leq n-1 .
$$

By combining the two inequalities we obtain that

$$
\left|\operatorname{sign}_{n}\left(\alpha_{n}^{p} \delta\right)-\overline{\operatorname{sign}}_{n}\left(\alpha_{n}^{p}\right)\right|=\left|\operatorname{sign}_{n}\left(\alpha_{n}^{p} \delta\right)+2 p\right| \leq 2 n-2
$$

for every integer $p \in \mathbf{Z}$. Recall that $\operatorname{sign}_{n}\left(\alpha^{p} \delta\right)=\left(\operatorname{sign} \circ \Psi_{n}\right)\left(\alpha^{p}\right)$. The above inequality then says that the restriction of the quasimorphism sign $\circ \Psi_{n}$ to the cyclic subgroup generated by $\alpha$ is within bounded distance from the homogeneous quasimorphism $\overline{\operatorname{sign}_{n}}$ restricted to the cyclic subgroup $\langle\alpha\rangle \subset \mathbf{B}_{n}$. This implies that sign $\circ \Psi_{n}$ is unbounded on the cyclic subgroup generated by $\alpha$ and, moreover, its homogenization restricted to $\langle\alpha\rangle$ is equal to $\overline{\operatorname{sign}_{n}}$. It is then a consequence of Corollary 1.9 that

$$
\operatorname{sg}_{4}\left(\Psi_{n}\left(\alpha^{p}\right)\right) \geq \frac{\left|\overline{\left(\overline{\operatorname{sign} \circ \Psi_{n}}\right)(\alpha)}\right|}{C_{\text {sign }}} \cdot p-D_{\Psi_{n}} \geq p-3 n+1
$$

\section{References}

[1] M Brandenbursky, On quasi-morphisms from knot and braid invariants, J. Knot Theory Ramifications 20 (2011) 1397-1417 MR2851716

[2] M Brandenbursky, J Kędra, Ś Gal, M Marcinkowski, Cancelation norm and the geometry of bi-invariant word metrics, Glasgow Math. J. (2015) published electronically

[3] D Burago, S Ivanov, L Polterovich, Conjugation-invariant norms on groups of geometric origin, from: "Groups of diffeomorphisms", (R Penner, D Kotschick, T Tsuboi, N Kawazumi, T Kitano, Y Mitsumatsu, editors), Adv. Stud. Pure Math. 52, Math. Soc. Japan, Tokyo (2008) 221-250 MR2509711

[4] D Calegari, scl, MSJ Memoirs 20, Math. Soc. Japan, Tokyo (2009) MR2527432

[5] J-M Gambaudo, É Ghys, Commutators and diffeomorphisms of surfaces, Ergodic Theory Dynam. Systems 24 (2004) 1591-1617 MR2104597

[6] E A Gorin, V J Lin, Algebraic equations with continuous coefficients, and certain questions of the algebraic theory of braids, Mat. Sb. 78 (1969) 579-610 MR0251712 In Russian; translated in Math. USSR-Sb. 7 (1969) 569-596 
[7] M Kawasaki, Relative quasimorphisms and stably unbounded norms on the group of symplectomorphisms of the eucledean spaces. To appear in J. Sympl. Geom.

[8] D Kotschick, Stable length in stable groups, from: "Groups of diffeomorphisms", (R Penner, D Kotschick, T Tsuboi, N Kawazumi, T Kitano, Y Mitsumatsu, editors), Adv. Stud. Pure Math. 52, Math. Soc. Japan (2008) 401-413 MR2509718

[9] C Livingston, A survey of classical knot concordance, from: "Handbook of knot theory”, (W Menasco, M Thistlethwaite, editors), Elsevier, Amsterdam (2005) 319-347 MR2179265

[10] C Livingston, The stable 4-genus of knots, Algebr. Geom. Topol. 10 (2010) 2191-2202 MR2745668

[11] D D Long, Strongly plus-amphicheiral knots are algebraically slice, Math. Proc. Cambridge Philos. Soc. 95 (1984) 309-312 MR735371

[12] H R Morton, Closed braids which are not prime knots, Math. Proc. Cambridge Philos. Soc. 86 (1979) 421-426 MR542687

[13] K Murasugi, On a certain numerical invariant of link types, Trans. Amer. Math. Soc. 117 (1965) 387-422 MR0171275

[14] A G Tristram, Some cobordism invariants for links, Proc. Cambridge Philos. Soc. 66 (1969) 251-264 MR0248854

MB: Department of Mathematics, Ben-Gurion University

Beer-Sheva, Israel

JK: Institute of Mathematics, University of Aberdeen

Aberdeen AB243UE, UK

Instytut Matematyki, Uniwersytet Szczeciǹski

70-451 Szczecin, Poland

brandens@math.bgu.ac.il, kedra@abdn.ac.uk

Received: 21 August $2014 \quad$ Revised: 9 February 2015 